C2020. Licensed under the Creative Commons Attribution-NonCommercial-NoDerivatives 4.0 International http://creativecommons.org/about/downloads

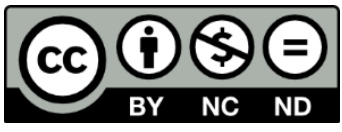

This is the accepted version of this article. The version of record is available at https://doi.org/10.1016/j.appet.2020.105040 


\title{
"A step in the journey to food waste: how and why mealtime surpluses become unwanted"
}

\author{
Accepted for publication in the journal Appetite \\ (November 2020)
}

\author{
Alia Aleshaiwi ${ }^{1}$ and Tim Harries ${ }^{2}$ \\ ${ }^{1}$ Kingston Business School, Kingston University London, Kingston Hill, Kingston- \\ Upon-Thames KT2 7LB \\ Al Imam Mohammad Ibn Saud Islamic University, Riyadh, Saudi Arabia, Postal \\ Code: 11432, P.O.Box: 5701 \\ akaleshaiwi@outlook.com \\ Corresponding author \\ ${ }^{2}$ Kingston Business School, Kingston University London, Kingston Hill, Kingston- \\ Upon-Thames KT2 7LB \\ t.harries@kingston.ac.uk
}




\section{Abstract}

This paper explores how and why Saudi householders designate mealtime leftovers as unwanted, thereby making them more likely to become waste. The paper argues that although over-provisioning is cited as one of the main antecedents for food waste, food becomes unwanted before it becomes waste and the designation of overprovisioned food as unwanted is an important but neglected driver of food waste. The study draws on in-depth interviews with 28 Saudi women to reveal four main reasons for the classification of leftovers as unwanted. First, food touched by others, such as plate leftovers, is perceived as unclean because it fosters feelings of disgust. The causes of this disgust are related to changes in social norms of eating. Second, clean leftovers are seen as less desirable for hedonistic reasons because they do not provide the same sensory eating experience as fresh food. Third, the rejection of leftovers might be related to the implications of rising levels of affluence for the attractiveness of leftovers. Lastly, food becomes unwanted as a result of social norms regarding eating home-cooked food outside the home. This highlights the possible influence of norms on the wider issue of food waste. These findings illustrate the circumstances in which food is categorized as unwanted and underline the significance of social and hedonistic factors. Such findings help us to better tackle the issue of food waste by providing in-depth insights into an important part of the journey between overprovisioning and food waste. The findings also strengthen the scarce literature on food waste in Arabic and other Islamic countries and highlight underlying normative and cultural aspects in such countries that are relevant to the issue of household food waste.

Keywords: Food waste, surplus food, leftovers, disgust, hedonism, affluence

\section{Introduction}

This paper explores the journey of meal leftovers in Saudi households and asks how they end up becoming unwanted. Although the rejection of surplus food is one of the 
main antecedents of food waste behavior (Clark and Manning, 2018, Porpino et al, 2016; Quested et al, 2013; Romani et al, 2018; Stancu et al, 2016), the food waste literature rarely pays much attention to the creation of surplus, treating it, rather, as just one part of the overall process of provisioning, storage and meal preparation (Andrews et al, 2018).

The seminal work on the creation of surplus food is Evans' $(2011,2012)$ UK ethnographic study into how and why households end up wasting food. Evans (2014) provides "a theoretical sketch" that explores the journey of food to waste. Relevant to this paper is Evans' remark regarding "the gap in disposal" in which he unpacks the process through which surplus food crosses the line to become waste. He points out that surplus food is rarely disposed of immediately. It enters a gap where ambiguities and anxieties surrounding its residual value render that food neither useful nor useless. The food is surplus to the immediate need, but still has potential value because it is edible. Most surplus that enters the disposal gap is first stored but not eaten within the timeframe required. As such, "the gap can be viewed as something that extends the process of ridding" (Evans, 2014: 54) and reduces anxieties about food waste (Evans, 2012), but without necessarily reducing waste.

Postponing the act of discarding leftovers until they become inedible is a key driver of food waste (Blichfeldt et al, 2015). While leftovers are sometimes stored with the intention of consuming them later (Cappellini and Parsons, 2012), the possibility of using leftovers decreases over the course of their short lifespan (Evans, 2014). This can be illustrated by examining how people handle leftovers. Previous research suggests that the value of leftovers is often ambiguous because they are not as desirable as fresh food (Blichfeldt et al, 2015; Evans, 2014; Waitt and Phillips, 2016). As such, the disposal of leftovers is often enacted via a two-stage process of storing then binning. After finishing a meal, surplus is stored in order to open up the possibility of later consumption. However, it usually deteriorates before it can be eaten, so is binned for reasons of taste and smell (Waitt and Phillips, 2016). Accordingly, procrastination over the use of leftovers is a key cause of food waste (Porpino et al, 2016; Blichfeldt et al, 2015). 
The successful consumption of leftovers depends, in part, on finding future useoccasions for that food (Hebrok and Heidenstrøm, 2019; Evans, 2014). This notion of classifying surplus not based on the intrinsic value of the different parts, but rather on their possible reuses and future meals plans, is also evident in Cappellini (2009) and Cappellini and Parsons' (2012) papers on meal leftovers. However, as pointed out by other authors (Andrews et al, 2018; Cappellini and Parsons, 2012; Evans, 2014; Romani et al, 2018; Watson and Meah, 2013), plans to eat or use surplus food in the future can be confounded by unexpected events.

Another set of factors that prevent food from becoming unwanted are thriftiness, household economics and a sense of responsibility (Graham-Rowe et al, 2014; Watson and Meah, 2013). Motivations to minimize waste by consuming surpluses seem to be rooted in the desire to save money (Lazell, 2016). Cappellini and Parsons (2012) point out that eating leftovers is seen as a thrift act, in terms of saving time and work in the kitchen and freeing up resources for future consumption. However, the eating of leftovers is a sacrifice that not every member of the family is willing to take; mothers often oblige themselves to eat leftovers so other family members can enjoy fresh food (Cappellini, 2009).

The relative unattractiveness of leftovers is one of the main reasons they become waste (Clark and Manning 2018; Porpino et al, 2016; Romani et al, 2018). With the exception of particularly "flavoursome leftovers" (Andrews et al, 2018) such as special food prepared for guests (Southerton and Yates, 2014), leftovers are often seen as less tasty than fresh food and, having appeared in a previous meal, as having lost their novelty (Cappellini, 2009). Surplus is also rejected because of the perceived health risks associated with eating food that is not fresh (Andrews et al, 2018; Farr-Wharton et al, 2014; Hebrok and Heidenstrøm, 2019; Soma, 2017) and because the "multiplicity of choices" available in some countries makes the consumption of leftovers less necessary (Porpino et al, 2016). 
Although the reasons uncovered in the above studies help explain how surplus becomes waste, they do not explain how food becomes unwanted in the first instance. It is important to note that our term "unwanted" is different in important regards to Evans' term, 'excess'. In Evans's study (2014: 65), excess "cannot be imagined as useful or valuable" and "the slip from surplus to excess marks the point at which food is no longer food"; as such, excess often ends up in the bin. In the present study, unwanted food refers to any surplus that families do not want to eat but consider valuable and not to be wasted. As this implies, food becomes unwanted before it becomes excess or waste. As suggested by the data collected in this study, unwanted food does not necessarily end up being wasted; in Saudi at least, households try to deploy its remaining value in a manner that respects the religious teaching of Islam.

The guidance of Islam states that Muslims should live their life in moderation and prohibits waste in every aspect of their life. References to the "waste" and Englishlanguage synonyms occur up to 50 times in translations of the Holy Qur'an (Yoreh and Scharper, 2020). Muslim participants in Soma's $(2016 ; 2017)$ study of food waste in Indonesia used the Quaranic term mubazir to relate to the sinful act of being wasteful. Another Quananic term, musrifün, refers to those who waste by extravagance:

"eat and drink, but waste not by extravagance, certainly He (Allāh) likes not AlMusrifūn" [The Noble Qur'an, Sūrat Al-An'ām, verse 31]

The former term (musrifūn) refers to waste in general, while the latter (mubazir) refers to the waste of money. In general, Muslims tend to use both terms to refer to waste and excessiveness (Yoreh and Scharper, 2020). In Islam, food is considered a blessing and a gift from God, so to waste food is a sin and act of ingratitude:

"Eat of the provision of your Lord, and be grateful to Him" [The Noble Qur'an, Sūrat Saba', verse 15]

Although the prohibition of wastefulness and excessive consumption is clear in the Qur'an and Sunna (prophet Muhammed's way of life, sayings and actions), a large amount of unwanted food and waste is generated in Islamic countries (COMCEC, 2017; Soma 2016; Soma; 2017; Yoreh and Scharper, 2020) including Saudi Arabia (COMCEC, 2017). The Saudi Grains Organization (2019) conducted a major study to establish a national food waste and loss baseline across the supply chain. The study 
reveals that about $19 \%$ of food purchased by households is wasted each year; this equates to 2.3 million tons of food - 105 kilograms of food per person.

Taking inspiration from previous studies, this paper seeks to contribute to the current food waste literature by focusing on how and why mealtime surpluses become unwanted in Saudi households. In doing so, we have chosen to focus exclusively on lunch, which in Saudi is the main meal of the day. In the UK, 33\% of all domestic food waste is generated at the main evening meal (WRAP, 2014) and in Saudi, the fact that one-third of purchased rice is wasted (Saudi Grains Organization, 2019) suggests that lunch, the main meal at which rice is consumed, is a significant source of waste here as well. Lunch is usually the only home-cooked meal that all members of Saudi families are expected to attend; it is the meal that requires most planning and preparation; it is the meal for which the bulk of groceries are bought, and, as pointed out by interviewees, it is the meal from which most surplus is generated. Furthermore, lunch is the only meal that is prepared almost every day in Saudi homes; that is served at a fixed time, and for which a certain set of dishes are prepared. The study of such a mundane and routinised meal is a good opportunity to reveal (ir)regularities, patterns, and taken-for-granted norms.

This paper aims to help ascertain the relevance to the global debate of cultural contexts outside of the Western World. The paper draws on fieldwork conducted in Saudi Arabia, an Arab and Islamic country. Apart from Soma's (2017) study of Indonesia, most previous research was conducted within a western cultural context. Little previous research has been conducted in Arab and/or Islamic countries and what research there is has often limited itself to the identification of attitudes, determinants and behaviors associated with food waste - such as poor planning and over-provisioning (e.g. Abdelradi, 2018; COMCEC, 2017; Mattar et al, 2018; SAGO, 2019). Whilst such studies added to the understanding of food waste behavior in Arab, Islamic areas, they do not address what happens in the stage between 'surplus' and 'waste' and how surplus is handled and rejected the household level. 
In addition, many existing studies present food waste as primarily a matter of individual action. We argue that approaches that focus on individuals as a unit of analysis underestimate the extent to which food consumption is embedded in social practices. As such, our enquiry into surplus generation in Saudi households is informed by social practice approaches: we examine the handling of surplus as a social rather than individual phenomena. A practice-based approach enables the analyst to examine individual behaviors within the social contexts that shape them (Delormier et al, 2009). Such an approach is consistent with the view that although practices are social, individuals retain some freedom in how they perform them (Schatzki 1996; Warde, 2005). In our study of how surplus becomes (un)wanted, we look at how people categorize surplus food within the context in which food practices are located.

This paper examines two types of surplus: food that is left on individual plates and food that is cooked but not served. This distinction will uncover how perceptions of cleanness and edibility, and feelings of disgust, impact the handling of surplus and the classification of food as unwanted. In the food waste literature, there is limited mention of plate leftovers and how that food is assessed and handled. However, in the UK around 1 million tons of plate leftovers - worth $£ 3.3$ billion - are wasted, making plate leftovers one of the main sources of domestic food waste (Wrap, 2008). In Saudi, a 2017 survey study estimated that $13 \%$ of food wasted in homes comes from plate leftovers (COMCEC, 2017). Accordingly, it is important to understand when and why plate leftovers become unwanted. In regard to the second type of surplus discussed in this paper, food that is cooked but not served, previous research has revealed how this form of surplus becomes waste as a result of the complex rhythms of everyday life and because of the two-stage process of food-handling mentioned earlier. Whilst the findings of such research are important, they do not address two of the questions tackled in this paper: how disgust sensitivity (section 3.1.) and seeking sensory eating experience (section 3.2.) influence whether surplus food becomes unwanted. This paper also addresses the influence of societal affluence and the availability of fresher alternatives on the attractiveness of leftovers (section 3.3.) Lastly, the paper highlights how useful surplus might become unwanted because of social norms that discourage eating leftovers outside the home (section 3.4.) 


\section{Methodology}

This paper reports one set of findings from a study that used qualitative methods to explore the journey of unwanted food in Saudi households. In Saudi, women usually handle most food-related tasks such as cooking and the management of surplus, with men helping with shopping and taking out unwanted food. Hence, all participants were females who were at least partially responsible for food in their households. A snowballing strategy was used as a means of establishing the trust necessary for the recruitment of these females, because this is a suitable method for gaining access to a population that is generally so reluctant to participate in research (Magnusson and Marecek, 2015) and it would have been impossible for the first author to recruit householders without first being introduced by someone they know. Recruitment involved asking friends and acquaintances to send the research invitation letter to people they know. The first author then was contacted by women who were interested in being interviewed and with whom she has no prior relationship. At the end of each interview, participants were given an invitation letter that they were asked to send to others who might be interested in participating in the research.

A total of 28 interviews were conducted between July 2018 and January 2019 in Riyadh, the capital of Saudi Arabia. Interviews lasted between half an hour and two hours and for 70 minutes on average. The interviews covered a range of topics related to food management at home and the food cycle from shopping to ridding - with particular focus on meal-times. A responsive interviewing style (Rubin and Rubin, 2012) was adopted as it was critical to establish trust by creating an atmosphere that was relaxed and friendly. Responsive interviewing is a style of qualitative in-depth interviewing that treats the interviewee more as a partner than as subject of research. It emphasizes the importance of building a relationship that is based on trust - for example, by reacting to what interviewees say and revealing the interviewer's emotions. This approach "will usually elicit better answers" than more neutral questioning (Rubin and Rubin, 2012: 165). As in all good quality interviewing, 
questions were kept open and it was made clear to interviewees that there were no expected answers, right answers or wrong answers. Responsive interviewing is adaptive and acknowledges each participant's unique experience; as such, although each interview covered the same topics, follow up questions and probes were devised during the interview.

To analyze the interviews, thematic analysis was applied (Braun and Clarke, 2013); a systematic, six-stage process of analysis aimed to identify themes and patterns of meaning across the data. This method was chosen because it enables the analyst to go beyond the semantic content of the data and identify underlying ideas and assumptions (Braun and Clarke, 2006) - thereby addressing the research aim of identifying underlying factors and meanings. The initial phase of the analysis involved multiple readings of the verbatim transcripts. These were then coded comprehensively, looking for chunks of data that were relevant to the research questions. This was followed by a search for patterns, variabilities and consistencies across the data set in an iterative process that took the analyst back and forth between transcripts, codes, and pattern. Themes then were identified at the manifest and latent level. At this stage, the literature was revisited to inform the final version of the analysis and the themes were reviewed to ensure they were grounded in the data and told a coherent story. The study was granted ethical approval by the Kingston University Research Ethics Committee. All participants signed informed consent forms.

\subsection{Participants}

All participants were female, and lived in Riyadh, the capital of Saudi Arabia. All were Saudi citizens. Amongst Saudi

\begin{tabular}{|c|c|c|c|c|c|c|c|c|}
\hline \multicolumn{9}{|c|}{ Table 1: Profile of participants } \\
\hline \multirow[t]{2}{*}{ Age } & $20 \mathrm{~s}$ & $30 \mathrm{~s}$ & $40 \mathrm{~s}$ & $50 \mathrm{~s}$ & $60 s$ & & & \\
\hline & 8 & 11 & 6 & 2 & 1 & & & \\
\hline \multirow{2}{*}{$\begin{array}{l}\text { Household } \\
\text { number }\end{array}$} & 2 & 3 & 4 & 5 & 6 & 7 & 8 & 9 \\
\hline & 2 & 6 & 8 & 2 & 4 & 2 & 1 & 3 \\
\hline \multicolumn{9}{|c|}{ Employment } \\
\hline & \multicolumn{4}{|c|}{ Work full day } & & \multicolumn{3}{|c|}{8} \\
\hline & \multicolumn{4}{|c|}{ Work half day } & & \multicolumn{3}{|c|}{14} \\
\hline
\end{tabular}

citizens, there is a fair degree of cultural and ethnic homogeneity (Metz, 1992; Nyrop, 
1997), with almost all being Muslims and nearly $90 \%$ being ethnic-Arabs (IOC, 2006). The relative cultural homogeneity of the citizenry indicates a homogeneity of values that is reflected in the triple foundation of Islam, family, and tradition (Nyrop, 1997) as well as in the common mother tongue of Arabic (Al-Seghayer, 2011).

A summary of participants' profiles can be seen in Table 1. Of the 28 interviewees, 22 worked either full- or half-time. In the past, working hours in Saudi were shorter and most jobs ended between $1 \mathrm{pm}$ and $3 \mathrm{pm}$, so lunch became established as the main meal of the day. While workers in the education sector work half days of five or six hours and finish at 1-2 p.m., those in the private sector or health sector usually work 8-hour a day that end at 4-5 p.m. These changes in working hours have affected meal timings. Our data suggests that although lunch is still the main home-cooked meal, it is only eaten after work or school have ended - even if this is as late as 7 p.m.

Households' economic situations were discussed during the interviews. Interviewees raised the topic when talking about their effort to save money and cut spending, the financial difficulties they faced, or when asserting that money was not a concern for them. In the analysis, we were interested in exploring whether households' perceived economic situation had an influence on the ways interviewees and their families handle surplus food. Our data suggests that norms and preferences are more important factors than economic situations as predictors of the classification of food as unwanted (see section 3.3). It is important to point out, however, that our sample did not include any participants who rely on food banks or receive social benefits.

\section{Findings and discussions}

\subsection{Inedible surplus: Cleanness boundaries and disgust}

This section focuses on how interviewees classify which elements of lunch surplus are clean and which are not. Surplus that is perceived as clean is often put in the fridge whereas unclean surplus is perceived as "disgusting" and therefore as inedible and unwanted. However, these boundaries of cleanness varied among interviewees, with 
more surplus being classified as unclean among families whose members eat from individual plates than among those that eat from communal plates.

Eating from communal plates and the use of hands to eat were usual in Saudi prior to the discovery of oil in the 1980's. With the discovery of oil and the resulting influx of foreign workers and growth in foreign travel and foreign television, Saudis had greater opportunity to learn about other cultures. As a result, new tastes were acquired and manners of eating started to change. The use of cutlery and individual plates began to replace eating by hand and from communal plates (Al-Othaimeen, 1991).

These changes in eating manners seem to relate to the heightening of food-related disgust observed in our data. Those interviewees that grew up using individual plates conceived of eating from someone else's plate as "disgusting" and classified food that had been touched by another person as not natheef (unclean) and untouched food as natheef (clean). In contrast, interviewees whose families continued to eat from communal plates did not use the phrase not natheef to describe surplus from plates and asserted an absence of disgust when sharing food with family. Falling between these two categories are families who mainly use individual plates and only eat from communal dishes when eating certain traditional meals; such families tended to classify less plate leftovers as unclean compared to the first group but more plate leftovers as unclean than the second group.

These differences are highlighted in the reports of people that grew up in families that ate from communal plates but married partners that grew up using individual plates. These differences in ways of eating and handling food surplus alerted them to feelings of disgust that they had not experienced when growing up. An example of this is Faouzia (40s- in work ${ }^{1}$ - mother of five children) who, unlike her husband, grew up in a family that only used communal plates. After a few years of marriage, she noticed that her children felt it was disgusting to eat from a communal plate. She therefore decided to serve food on a communal plate in order to teach her children to be one

\footnotetext{
1 The term "in work" refers to women who work outside the home for income
} 
"united" family: "I feel we are a family, so I don't like the attitude, 'don't touch my plate'. I like togetherness". As a result of this instructional practice, Faouzia's family do not have unclean plate leftovers; lunch leftovers are generally kept in the fridge and eaten later without feelings of disgust.

Qamar (20s- in work- mother of a toddler), who also grew up eating from a communal plate but married a man whose family used individual plates, recalled an awkward encounter during a visit to her in-laws:

"I had food on my plate and I did not want to throw it away because I did not know what they would do with it. So I gave it to my husband and said 'eat it' (laughs). They opened their eyes wide as if to say, 'give him from the clean food'" Qamar felt anxious about her plate leftovers being thrown away. However, her inlaws, she explained, felt it was "disgusting" to share touched plate leftovers - even between husband and wife. Such food was seen as having been contaminated by the other person's spoon or hand, so as an unacceptable offering to another person.

The data suggests that families that only occasionally eat from communal plates have different perceptions of cleanness from those that do so regularly. When Somaya's (30s- in work- single and lives with her parents) family eat from a communal plate, food that is likely to have been touched is treated as unclean ("no one would accept it after your hand"), but food in the middle of the communal plate is considered clean and put back in the fridge. Suaad (40s- in work- mother of four children), whose family eat only the traditional kabsah (red rice) dish from a communal plate, does not keep any kabsah plate leftovers when her family eat from one plate because food that has been touched by others and mixed with side dishes "is not the same as when you serve it". Despite eating food from communal plates, these families consider it unacceptable and disgusting to eat food that has been touched by others while on the plate.

A third category of family provides an individual plate for each family member so they can use communal spoons to put food on their own plates and keep the rest of the food clean. Rana (20s- in work- single and lives with her parents) and Yara (20s- in work- mother of a toddler) pointed out that using individual plates makes handling surplus food straightforward: plate leftovers are automatically unwanted because 
they are considered unclean, messy, and unpalatable, while food in the main pots is put back in the fridge. However, the disgust sensitivity seems to depend on how food was touched and by whom. Fariydah (50s- housewife - mother and grandmother), Fatema (40s- in work, mother of six children), and Muneera (60s - mother and grandmother) put plated items of food (e.g. zucchini or pastries) back with the clean food if they are fairly certain that the items remained untouched by diners' fingers and spoons. However, they do not classify plated rice on individual plates as clean because it is difficult to assess whether it has been touched. For Nihad (40s- in work, mother of two children) and Aljazi (40s- in work- mother of seven children), whether a plate leftover is considered clean depends on who ate from that plate: Nihad does not mind eating her children's plate leftovers, but will not eat those of other relatives or strangers; Aljazi eats leftovers from the plates of her older children but not from those of the younger, messier eaters.

Few studies in the food waste literature have directly addressed the handling of different types of plate leftovers. Evans (2014) points out that, unlike food that is not served, served food is often removed and does not enter the gap of disposal discussed earlier (section 1). Andrews et al (2018) refer to such leftovers as "used or secondhand" and point out that, though they are edible, people are unwilling to eat them because they are "unappealing" and may have been "slobbered on". Our study goes further and extends previous findings by contributing towards a better understanding of the underlying reasons for the rejection of plate leftovers. It highlights the cultural significance of sharing food and the role of disgust in classifying plate leftovers as clean/unclean, thereby wanted/unwanted. Such classification involves the rejection and removal of inappropriate elements that are classified as (in the terms of Douglas, 2002) dirt. Dirt, as Douglas (2002) argues, is matter "out of place" and by-product of a classification that defines what is dirty or clean. This section has revealed key insights into the classification of what is clean surplus and the consequent rejection of what is unclean.

Our study also examines the visceral elements of surplus handling practices. Previous research highlighted the role of the visceral response of disgust in the cultural 
classification of food as waste (Waitt and Phillips, 2016; Watson and Meah, 2013). Visceral disgust can be fostered by affective experience or the physical deterioration of matter (Waitt and Phillips, 2016; Watson and Meah, 2013). In both cases, disgust draws the boundaries between categories of clean and dirty. In this study, the variations in disgust sensitivity and perceived edibility of plate leftovers among the three categories of families suggest that disgust is socially learned rather than biologically based (see Blichfeldt et. al, 2015; Graham-Rowe et al, 2014; Waitt and Phillips, 2016). For example, before their mother intervened, Faouzia's children had accepted their father's view that sharing food is disgusting. Returning to the changes Saudi Arabia has witnessed, it seems that these cultural variations between the three categories could be related to the adoption of foreign manners of eating. Those interviewees who still used a communal plate, such as Qamar and Faouzia, felt that they had strong bonds with their families and so did not mind sharing food with them. Other interviewees felt that it was neither acceptable nor palatable to eat food touched by another diner. Accordingly, keeping the tradition of eating from a communal plate or adopting new manners of eating have implications for which food is considered disgusting, unclean and, therefore, unwanted.

Disgust, we argue, operates as a rejection system. It is defined as "revulsion at the prospect of (oral) incorporation of an offensive object" (Rozin and Fallon, 1987: 23), where the offensive objects are "contaminants" (Rozin and Fallon, 1987) that are perceived as dangerous or dirty (Waitt and Phillips, 2016). In this study, 'the offensive objects' are conveyed be the touch of a hand or spoon and rejection is on the basis that these render food unsuitable for consumption, even if not necessarily dangerous (Andrews et al, 2018). Disgust makes people step back and draw a protective line between the self and the threat (Haidt et al, 1997) and that line functions as a rejection system to separate the edible from the inedible. However, the ways families in this study dealt with plate leftovers suggest that there is no universal, clear or fixed line of edibility (Waitt and Phillips, 2016; Watson and Meah, 2013); rather, edibility is related to cultural factors as well as biological ones (Blichfeldt et. al, 2015). The visceral response of disgust, our data suggests, is not primarily triggered by the sensory properties of food, such as smell or taste, but by ideational concerns about who 
touched it (Haidt et al, 1997; Rozin and Fallon, 1987). As such, the ways in which interviewees draw the virtual line of edibility suggest that disgust is a socially constructed concept that evolves through changing patterns of socialization.

\subsection{Edible surplus: hedonism and sensory experience}

This section explores how 'clean' lunch leftovers become unwanted after they have been put away for future use. Amongst Saudis, cooked food that is kept for at least one night is known as 'bayt'. Food commonly moves from being 'bayt' to being 'unwanted' for hedonistic reasons related to sensory experience. In this study, sensory experience is related to two hedonistic factors: the sensory properties of food, such as taste, texture, smell and sensory variations which refer to the desire to avoid eating the same food on consecutive days.

\subsubsection{Sensory properties and variations}

For some interviewees, re-using and transforming bayt food is enjoyable and fulfilling if its sensory properties have not been compromised. For these interviewees, the sensory properties of taste, texture and smell are key when deciding whether to use bayt food. Fariydah enjoys cooking and creating new dishes from bayt food: "If you open my fridge, you would see variety of things but I use all of them". She uses chicken leftovers to make shawarma or soups and lamb leftovers to fill samosa, but does not use leftovers whose texture has changed and are perceived as no longer appetizing. In the latter cases, the pleasure from reusing bayt food is outweighed by disadvantages of eating, and serving to her family, unappetizing food. A common example in the data of bayt food losing its appeal are the traditional meals of jareesh and qurasan. Jareesh is crushed wheat mixed with yoghurt and qurasan is wheat-based bread saturated with gravy. Most often, these dishes become mushy and lose their texture and taste the day after they have been prepared, so are deemed unwanted even though they are safe to eat. As used by interviewees, the adjective 'bayt' has a negative connotation; it describes food that has lost some of its original sensory properties. For example, Hayla (50s- 
housewife - mother and grandmother) explained how she adds other ingredients to bayt food to transform it to a new meal that "does not taste bayt". She only keeps bayt food that can be transformed without sacrificing its sensory properties; she re-uses rice that is a few days old because it can be flavored with other ingredients, but she does not re-use meats and vegetables because they become flavorless, their smell changes and "it is obvious they are bayt". Therefore, food becomes unwanted through surplus classification practices, rather than innate material qualities (Cappellini 2009; Cappellini and Parsons, 2012; Waitt and Phillips, 2016). The ways Fariydah and Halya classify leftovers suggest, as Evans (2011) points out, that there is nothing careless about the acts of handling surplus. Before bayt food becomes unwanted, it is handled with care to assess the possibility of making it less bayt. If that is not possible, bayt food becomes unwanted and is replaced with food that interviewees enjoy cooking and serving to their families.

As well as being deemed unwanted because of loss of sensory properties, leftovers are sometimes deemed unwanted because of the desire to avoid eating the same food on consecutive days. It is considered "boring" to eat bayt food because this would be to repeat a recent meal and the food would have lost its novelty. For example, Hanan (20s- in work- mother of two children) and Walaa (40s- housewife- mother of two children) felt they had to serve a fresh lunch every day because their husbands did not like "repetition" (Walaa) and would feel "bored" (Hanan). Similarly, Nihad argues that her family should be provided with a different meal each day because eating should be joyful. Previous studies recognise that the taste for variety in food consumption means that some people are not willing to eat the same food more than once within the timeframe that the leftovers command (Cappellini, 2009; Evans, 2011; Urrutia et al, 2019). This paper highlights the significance of food enjoyment in the unwillingness to repeat the same food on consecutive days. The Interviewees Nihad, Walaa and Hanan argue that variety in the type of meals served is an essential determinant of food enjoyment. For this reason bayt food usually becomes unwanted.

\subsubsection{Between Duty and Desire}


Although bayt food is perceived as less desirable, it is considered edible and so it is haram (forbidden) to waste it. Some interviewees communicated their dilemma over whether to maximize their families' enjoyment by serving fresh food every day, or to perform their perceived duty as Muslims by reusing edible bayt food. Interviewees condemned the act of wasting food and described such waste as "haram", "abhorred", and "a sin". As mentioned earlier, food is seen as a blessing, so to throw away food is a sin and act of ingratitude. To escape the sin of waste, some interviewees reported passing unwanted food to their house staff or forcing themselves to eat it. For example, Nihad offers the unwanted leftovers to the house staff and if the staff do not want them, she avoids committing the sin of waste by forcing herself to eat the leftovers. Another example that illustrates the struggle between duty and hedonism is Modhi (40s- in work- mother of five children). She described the leftovers in her fridge as lentils, liver, rice and fish. The lentil and liver were her husband's breakfast leftovers and the rice and the fish were left over from lunch on the previous day. She complained that her children did not want any of these leftovers, as they preferred something more appetizing:

"there is no cooperation at home. No one preserves. [...] for example I told you there was a bit of rice and fish left from yesterday... 'Ok... Instead of ordering a takeaway... you have not had lunch; instead of ordering from outside, eat what is in there.' They have not eaten all day. (But my children said) 'No, no we want this'. (My daughter) wanted fried chicken [...] the boy wanted shawarma. 'No, we don't want to eat [the leftovers]"

Modhi initially wanted to order a takeaway with her children but changed her mind when she opened the fridge and saw all the bayt foods. She threw away the lentils because they had gone bad, did not want to eat the fish and rice, and took few bites of the liver and put it back in the fridge:

Modhi: I put the liver in the fridge. I don't know; I might throw it away today. Interviewer: Why did you put it in the fridge?

Modhi: I felt it is a loss to throw it away: Haram; it is new. "Let me take it out." I ate a little. I ate two pieces for that not to be haram. You would say "why it is haram" (laughs) but for that not to be haram... let me take two pieces for that 
not to be haram and I put the rest in the fridge.

Interviewer: While you were putting it in the fridge, did you expect that it would be eaten the next day?

Modhi: I said, "my husband might eat it in the morning. He might see it. He might feel sorry for it and eat it" (laughs). Sometimes you see the blessing in the fridge saying to you "eat me eat me" (laughs) but you don't eat it.

$[\ldots]$

Modhi: I said I will eat the liver (laughs) and I won't buy fries and throw away the food that already is there. I mean at least if I throw away the leftovers that I have (after eating few bites), I won't be sinning. I ate something.

Modhi's story exemplifies the dilemma between sin and desire. She, like her children, wanted the more appetizing option, but by forgoing that option, eating a few bites of the unwanted bayt food and only throwing some of it away, she hopes to avoid sin. In addition, by taking a few bites and placing the rest back in the fridge, she keeps open the possibility of eating that food and thereby also escapes the sin of wasting edible food. As the stories of Nihad and Modhi illustrate, the waste of unwanted food is not taken lightly; to avoid the sin and the guilt of throwing away edible food, it is sometimes eaten joylessly or placed in the fridge until no longer edible.

The idea that good food should not be wasted was reported by participants of previous food waste studies. Studies conducted in the UK (Graham-Rowe et al, 2014; Evans, 2012), Australia (Waitt and Phillips, 2016), the US (Porpino et al; 2015), Denmark (Blichfeldt et. al, 2015) and Norway (Hebrok and Heidenstrøm, 2019) reported that none of their participants viewed food waste positively or were carefree about the acts of binning. Therefore, unwanted surplus is often placed in the fridge and enter the disposal gap until it becomes no longer edible and so the uncomfortable feeling of wasting is reduced (see Blichfeldt et al, 2015; Evans, 2014; Hebrok and Heidenstrøm, 2019). However, in this study of Saudi households, most unwanted food does not follow the trajectory noted in previous studies. Our data suggests that, even if the food is initially placed in the fridge, unwanted food often ends up outside the waste stream (Modhi, for example, passes most of the unwanted food to animals). 
Although this goes beyond the topic of this paper, it is worth pointing out that deeming food as unwanted is not an end point, but can begin a complex trajectory that ends in directing unwanted food to a variety of routes outside the waste stream. In this way, our interviewees were able, to some extent, to negotiate the tension between desire and duty. This issue will be addressed more fully in future work.

\subsection{3. (less) ordinary meals}

It is instructive to compare quotidian homemade lunches to less ordinary meals such as restaurant meals and food made during the month of Ramadan. Such a comparison further illustrates the significance of sensory properties and variations in the generation of unwanted food.

Of relevance to the use of bayt food is the inter-generational difference, amongst the families in this study, in preferences for restaurant food compared to home-cooked food. The introduction and the expansion of fast food establishments and restaurants are a relatively recent phenomenon in Saudi and resulted from the oil-fueled economic boom (Hamdan, 1990). Today, restaurants and food courts are common in Saudi high streets and malls, and ordering takeaway is becoming easier with the availability of home delivery services. Our interview data suggests that younger people have a greater taste for outside/restaurant foods than older people, who tend to prefer home-cooked meals.

Outside food seems to provide an enhanced sensory experience for younger generations. When Muneera re-heats lunch leftovers for her family, her grandchildren sometimes order a takeaway instead. Muneera explained this by saying that "youngsters [...] want combo meals (a sandwich and fries)" while she, a representative of the older generation, does not like "these stuff". Similarly, Ghada (30s- in work - mother of two children) complained that her six-year-old and thirteen-year-old children prefer fast-food such as pizza or burgers over "the routine food" she cooks. What Muneera and Ghada complain about suggests a generational difference in perceptions of the sensory properties of home-cooked 
and outside foods. As a result, outside food is preferred over bayt food by younger people and bayt food is more likely to become unwanted.

Preference for restaurant food is also common among the young couples in our sample, who even set aside their dislike of bayt when it concerns takeaway food. Ashjan (in her 30s - work - wife) and Qamar both reported that their husbands prefer the taste of restaurant food, whether fresh or bayt, to that of home-cooked food. Ashjan explained that while her freshly cooked food might be comparable to outside food, it does not keep its taste the next day as well as outside food does. Qamar pointed out that compared to restaurant foods, her lunch is "ok" but not "brilliant" and that when re-heated, "it is not like the fresh one that is just cooked. It is not the same quality". In Qamar and Ashjan's households, outside bayt food is eaten joyfully, but bayt home-cooked food is deemed unwanted. The taste of outside food seems to bring pleasure and joy. For example, Rana said that she loves fast food and described it as irresistible: "the day I have dinner from outside, I feel happy (laughs), like a child". Although these interviewees enjoy restaurant foods, home-cooked meals are appreciated in their households because they are healthier and more wholesome. Restaurant food, in contrast, is seen as a treat: an occasional indulgence rather than a staple. Therefore, when households bring food from outside, leftovers are highly "valued", as Ashjan mentioned, and enjoyed the following day. Outside food, as such, provides higher sensory experience compared to home-cooked food, so any bayt from the latter is more likely to become unwanted.

Before concluding this section, we will explain why lunch is kept overnight to become bayt, rather than eaten later in the same day. This could be illustrated by comparing main meals in the month of Ramadan with those eaten during the rest of the year. During Ramadan, many Muslims fast from dawn until sunset and, instead of lunch, eat iftar, the main meal eaten after sunset. Our data suggests that less surplus food becomes bayt and unwanted in the meal iftar, in comparison to lunch. This is for two main reasons. First, iftar dishes are described by interviewees as lighter than lunch dishes, and therefore as suitable to be eaten at any time of 
the day or night (as suggested by Zaineb: 30s- in work- single and lives with her parents) and are suitable to be "nibble[d]" (Amani: 30s- in work- single and lives with her parents). Lunch leftovers, on the other hand, are described as heavy and are usually not eaten as a snack or late at night for dinner because "you do not fancy lunch at night" (Hanan). Therefore, food left after lunch is usually kept to the next day, by which time it is categorised as bayt and is vulnerable to becoming unwanted.

The second reason for less iftar food becoming bayt is related to food novelty. Iftar dishes are described as novel and particularly appetizing and, as such, iftar leftovers are often eaten the same day as the original meal. Qamar has less bayt food in Ramadan because she prepares "innovative" dishes that her husband loves and she rarely makes the type of "rice and pasta" dishes cooked for lunch on "typical days". This is because "the atmosphere in Ramadan is different" and "one got used" to making new dishes. In addition, Qamar pointed out that cooking one meal for lunch fits neatly with her busy schedule but that during Ramadan she has more time to search for and make new and special recipes. As pointed out by Waitt and Phillips (2016), it is important to understand food practices within everyday household routines and rhythms. During Ramadan it is both socially normal and feasible to prepare a variety of new and appetizing dishes that enhance sensory variation and perceived sensory properties. Hence, iftar dishes are less likely to become bayt and unwanted than meals prepared outside of Ramadan.

Previous research suggests that less surplus is usually generated by special meals than by every-day meals (Cappellini and Parsons, 2012) and that leftovers from such occasions are more likely to be consumed (Southerton and Yates, 2014). Our study supports these findings by showing how surpluses from iftar and outside food are considered special, novel and tastier - and hence, as worth saving. Taste, being an important influence on waste (Andrews et al, 2018; Graham-Rowe et al, 2014; Southerton and Yates, 2014), is one explanation for less waste resulting from special meals. Our study explores generational differences in perceptions of the sensory properties of food and the implications of that for the classification of food as 
(un)wanted. In addition, by comparing ordinary and less ordinary meal occasions, this study helps explain when surplus is consumed the same day and when it is kept overnight to become bayt. Ordinary lunch meals are considered less exciting and hence are more likely to become bayt than special meals that are consumed less often. The study also addresses the reasons why these more appetizing and novel dishes are not served more often. Those dishes that are less likely to become unwanted are perceived as less wholesome and healthy (as with outside food) and as requiring the investment of a degree of time and effort that would disrupt the rhythms of everyday life (as with iftar). Our findings illustrate the importance of moving beyond individual choices to examine food practices within the contexts in which they are located.

Overall, this section contributes to the food waste literature and towards understanding the sensory dimensions of household surplus handling practices and the implication of these on the classification of surplus as (un)wanted. Our findings illustrate the role of sensory properties and variations in enhancing the sensory eating experience. The repetition of any experience is said to diminish the pleasure it produces (Alba and Williams, 2013) and the sensory properties of food is enhanced if the same dish has not been served recently (Andersen and Hyldig 2015; Rolls, 1986). This suggests that sensory variation enhances the perceived sensory properties of food and offers one explanation for why bayt food is often categorized as unwanted.

\subsection{Societal affluence and the attractiveness of leftovers}

The previous section highlighted the importance of sensory properties and variations on the categorization of surplus food as (un)wanted. This section focuses on the influence of societal affluence on the generation and attractiveness of leftovers.

The discovery of oil in Saudi in the 1980's marked a rapid shift in all aspects of life: food became more affordable, and new types of food outlets, such as supermarkets 
and restaurants, arrived and expanded in numbers. Most Saudi citizens became more affluent and having surplus became culturally normative.

Affluence in Saudi has impacted both surplus generation and the attractiveness of leftovers. Growing up, Muneera (now in her 60s) used to have limited access to food: "we used not to have these blessings. She (her mother) put a communal plate. If there was a meat or they put fenugreek to flavor foods; A plate of markook (a traditional dish). And we sat all together. Nothing stayed [i.e. there were no leftovers]".

In her childhood, her family made use of whatever food they had available. Now, on the other hand, she reported that her children and grandchildren often refuse to eat bayt food:

"It is a clean blessing. Nothing wrong with it [...] A blessing, thank God, but from the abundance of the blessing (her children say) 'we don't want re-heated food' (in a mocking tone) [...] They don't want bayt food because of the abundance of the blessing. But for those in need, it is like it has been just cooked" Muneera's present family do not need to eat bayt food out of necessity, so have the luxury of enjoying takeaways or fresh food instead. This suggests that the rejection of leftovers for the hedonistic reasons mentioned earlier (section 3.2) might be related to prosperity.

Similarly, Yara, in her 30s, pointed out that her mother's generation want to enjoy food and are less worried about surplus than that of her grandmother:

Yara: I feel the generation - not my grandmother generation; she was old - I mean the generation who had lived those days when the blessings entered... Interviewer: The [economic] boom? Yara: The boom. That generation have a problem [...] They have not experienced hunger like the generation before.

She further explained that people like her mother tend to overprovision food and do not want to be "frugal" because they consider food as a "blessing" that is to be enjoyed. Yara and Muneera's accounts suggest that the preference for abundance and 
the multiplicity of choices, having the option not to consume leftovers, are related to the rise of affluence. Hanan also felt that the affluence encourages Saudi citizens to enjoy food in abundance and not worry about money:

Hanan: I consider the Saudi nation, for me, as very profligate; we don't appreciate the blessing. Although my family do not throw anything away; at the same time, I say my family do not appreciate the blessing because they make extra.

Interviewer: Tell me more about this.

Hanan: I mean, I feel that there is a little bit of profligacy with food. There is affluence. We have affluence in the Saudi society. There is great affluence. [...] People, they say, "it is ok, it is ok. let's just eat".

For Hanan, Saudi citizens are mainly concerned with enjoying food and not with surplus generation or being economical. However, she and Yara do not suggest a total lack of concern about food waste; as they explained, their mothers are keen on not throwing away any food, despite the routinely generated surplus at their households. Interviewees clearly expressed their concerns about food waste but it seems that these concerns are more prominent after the generation of surplus than during its generation, when the desire to enjoy food and serve attractive food are more prominent. Less variety and volume of food, by suggesting frugality, can prevent families from enjoying the blessing of food.

Another point to add is that the changes to social norms prompted by the rise in affluence also seem to impact Saudi citizens with a lesser share in that affluence. Social norms, as our data suggests, are more important than economic status in the rejection of surplus food. In Saudi, eating flavourless food was once common (as Muneera's story illustrates); today, however, it is no longer the norm to eat and serve such food - even amongst less affluent interviewees. For example, Lama's late mother used to freeze rice and serve it during the week but, as Lama pointed out, people now consider such food as inedible. She (20s- housewife- mother of a toddler) tries to follow her mother's footsteps by reusing surplus because "life is hard" financially. However, despite income constraints, she and her husband sometimes "do not fancy 
eating" leftovers and eat something else instead. Another example is Aljazi, who occasionally has no money to buy food but who nevertheless often throws away leftovers because her children refuse to eat them. Similarly, although Fatema's "budget is bad" and "limited", her family sometimes "do not fancy" bayt food and eventually throw it away.

Our findings contribute to the literature that on food waste among those with limited income. There is no consensus on the relationship between income and food waste. While some quantitative studies found a link between these two factors (e.g. Abdelradi, 2018- Egypt; Gaiani et al, 2018- Italy), others found no statistical relationship between them (e.g. Koivupuro et al, 2012- Finland; Williams et al, 2012Sweden). Qualitative research by Porpino et al $(2015 ; 2016)$ in Brazil and the US supports the latter findings by suggesting that over-provisioning and an unwillingness to consume leftovers are the main reasons for food waste among lower-income households. Our data indicates that, in order to make sense of the apparent contradiction of surplus generation and rejection in households with limited income, it is important to recognize the social contexts of food practices. In this study, the practices of reusing surplus are influenced by the social norms that dictate what is acceptable to eat and serve and that are important for both affluent and less affluent interviewees.

The examples in this section illustrate the influence of societal affluence on both the generation and the attractiveness of leftovers. They show that the desire to eat and serve desirable food takes precedence over concerns about surplus generation. This demonstrates the significance of both affluence and hedonism (section 3.2) on the overall generation of surplus and the attractiveness of leftovers.

\subsection{The norm of taking inside food outside}

Interviewees reported that when plans for eating lunch are disrupted by the family taking their meal outside the home, the home-cooked food that had been intended 
for lunch is categorized as unwanted. These implications of the arrhythmic of everyday life were recognized in previous research (Andrews et al, 2018; Cappellini and Parsons, 2012; Evans, 2014; Romani et al, 2018; Watson and Meah, 2013).

One solution could be taking and consuming the home-cooked lunch to wherever the family is going. However, interviewees comments suggest that this would be to break social norms. In comparing her previous life in an English-speaking country to her current life in Saudi, Ghada noticed that other nationalities "don't rely on restaurants" like Saudis do and instead bring cooked lunch and leftovers from home. Having first "imitated them" in this regard, on returning to Saudi she felt embarrassed about bringing home-cooked food to work, because she felt people would "criticise" this behaviour and label it as "strange". The reason, as Ghada explained, is that the society is "lazy"; people "mock" those who go to the trouble of bringing and reheating leftovers at work and assume that such efforts are unnecessary when one can order a takeaway instead. They would say: "do you have nothing to do! Oh sister, buy from a restaurant, buy".

Interviewees argued that any attempt to defy this social norm and take home-cooked lunch outside the home might have negative consequences. For example, Sharifa (30sin work - mother of three children) used to give her son lunch leftovers to eat in his nursey but that this made him feel "embarrassed" and exposed him to "bullying" when he went up to elementary school. Sharifa herself had faced bullying at work on this issue. She described how an erstwhile colleague would make negative comments every time Sharifa brought lunch leftovers from home rather than eating the usual work-fare, take-away or sandwiches:

"it is the culture. You have to eat bread [...] for example if [her colleague] saw you eat anything wrong [she would say mockingly] 'oh here is the healthy one'."

The fear and embarrassment of being criticized or bullied can deter some people from taking lunch leftovers to eat outside the home. Even though Sharifa now feels "comfortable" about taking her home-cooked lunch to work, it is still only "tolerated" rather than fully accepted. The only food that Sharifa and Ghada feel it is culturally acceptable to bring from home to work or school is sandwiches. A possible reason 
could be that sandwiches do not need heating (as Ghada pointed out) and are considered a light snack rather than a lunch - which, as the data suggests, is a family meal that should be eaten at home. Although Sharifa brings leftovers to work, they are only enough to tide her over until she comes back home and cooks her family a proper lunch. As such, food that is eaten at work seems to be typically considered a casual meal that requires minimal preparation; therefore, it is "strange" for someone to eat lunch dishes outside the home. Accordingly, considering the norm of not taking inside lunch food outside, it is less likely for leftovers to be eaten outside the home when plans for eating them are interrupted by absence from the family home. Leftovers then become bayt and possibly unwanted, given the hedonistic factors discussed earlier (section 3.2.).

While the issue of taking food outside home might not be shared by all cultures, previous studies have highlighted the influence of other social norms on the generation of food waste. In the UK, for example, the sharing of unwanted food is limited by cultural norms around the acceptable sourcing of food (Lazell, 2016) and the perceived risk that this will open up a householders' taste in food to scrutiny (Evans, 2012). In Indonesia, the norm is for upper-income households to only gift each other food that is new, so unwanted leftovers are either given to the poor or stored and then thrown away (Soma, 2017).

\section{Conclusion}

By focusing on how mealtime surplus food becomes unwanted, this paper takes us one step closer to understanding the complex nature of household food waste. The findings extend the food waste literature in three ways. First, they highlight the importance of the stage in the journey that takes food from surplus to unwanted, and demonstrate that food becomes unwanted before it becomes excess and is wasted. Second, in the food waste literature, most accounts of food waste attribute this phenomenon to over-provisioning. This paper argues that an often-overlooked reason for food becoming unwanted at the household level is the rejection of over- 
provisioned foods rather than over-provisioning itself. The circumstances in which food is rejected and deemed unwanted underline the significance of social norms and hedonistic factors. These norms and factors are related to wider societal changes and shifts in eating preferences. In Saudi, these changes impacted on: factors that provoke disgust; the desire for out-of-home food, and the availability and affordability of fresh foods. Therefore, leftovers sometimes become unwanted and are replaced by fresh food that delivers superior sensory experience. Additionally, the social norms around taking home-cooked meals outside the home help push surplus across the line that differentiates 'useful' from 'unwanted' surplus. Third, the rapidity with which Saudi culture has changed since the 1980's, and the opportunity for local people to interact with other cultures, expose norms and normative changes that are less visible in more static societies.

If households' reasons for rejecting surplus are not taken into consideration, efforts to convince them to re-circulate and re-use surplus food are likely to be unsuccessful. We demonstrate that those wishing to encourage the consumption of surplus food and limit food waste would do well to better understand the reasons underlying householders' designation of surplus as unwanted and to acknowledge the importance of this stage of the journey taken by food in the domestic setting.

\section{Acknowledgements}

We are very grateful to the editor and anonymous reviewers for their helpful commentaries, each of which served to improve this paper.

\section{Authors contribution}

Under the supervision of the second author, the first author designed and carried out the study, including data collection, data analysis and the writing process. The second author participated in the writing process and critical review. Both authors have approved the final manuscript. 


\section{Declarations of interest}

None.

\section{References}

Abdelradi, F. (2018). Food waste behaviour at the household level: A conceptual framework. Waste management. 71, 485-493.

Alba, J. W., \& Williams, E. F. (2013). Pleasure principles: A review of research on hedonic consumption. Journal of consumer psychology. 23(1), 2-18.

Al-Othaimeen, A. (1991). Food Habits, Nutritional Status and Disease Patterns in Saudi Arabia. Doctoral thesis. University of Surrey (United Kingdom).

Al-Seghayer, K. (2011). English teaching in Saudi Arabia: Status, issues, and challenges. Hala. Andersen, B. V., \& Hyldig, G. (2015). Consumers' view on determinants to food satisfaction. A qualitative approach. Appetite. 95, 9-16.

Andrews, L., Kerr, G., Pearson, D., \& Mirosa, M. (2018). The attributes of leftovers and higher-order personal values. British Food Journal. 120(9), pp. 1965-1979.

Blichfeldt, B. S., Mikkelsen, M., \& Gram, M. (2015). When it stops being food: The edibility, ideology, procrastination, objectification and internalization of household food waste. Food, Culture and Society.18 (1), pp. 89-105.

Braun, V., \& Clarke, V. (2006). Using thematic analysis in psychology. Qualitative Research in Psychology. 3 (2), pp. 77-101.

Braun, V., \& Clarke, V. (2013). Successful Qualitative Research. A practical guide for beginners. Sage. Cappellini, B., (2009). The sacrifice of re-use: the travels of leftovers and family relations. Journal of Consumer Behaviour. 8 (6), 365-375.

Cappellini, B. \& Parsons, E., (2012). Practising thrift at dinnertime: Mealtime leftovers, sacrifice and family membership. The Sociological Review. 60(S2), pp.121-134.

Clark, J., \& Manning, L. (2018). What are the factors that an opportunity sample of UK students insinuate as being associated with their wastage of food in the home setting?. Resources,

Conservation and Recycling. 130, 20-30.

COMCEC, Standing Committee for Economic and Commercial Cooperation of the Organization of the Islamic Cooperation (2017). Reducing Food Waste in the OIC Countries. Turkey.

Delormier, T., Frohlich, K. L., \& Potvin, L. (2009). Food and eating as social practice-understanding eating patterns as social phenomena and implications for public health. Sociology of health \& illness, 31(2), 215-228.

Douglas, M. (2002). Purity and danger: An analysis of concept of pollution and taboo. Routlege. Evans, D. (2011). Beyond the Throwaway Society: Ordinary Domestic Practice and a Sociological Approach to Household Food Waste. Sociology. 46 (1), pp. 41-56.

Evans, D. ( 2012). Binning, gifting and recovery: the conduits of disposal in household food consumption. Environ. Plan. D Soc. Space. 30, 1123-1137.

Evans, D. (2014). Food waste: Home consumption, material culture and everyday life. Bloomsbury Publishing.

Farr-Wharton, G., Foth, M., \& Choi, J. H. J. (2014). Identifying factors that promote consumer behaviours causing expired domestic food waste. Journal of Consumer Behaviour. 13(6), 393-402.

Gaiani, S., Caldeira, S., Adorno, V., Segrè, A., \& Vittuari, M. (2018). Food wasters: Profiling consumers' attitude to waste food in Italy. Waste Management, 72, 17-24.

Graham-Rowe, E., Jessop, D. C. \& Sparks, P. (2014). Identifying motivations and barriers to minimising household food waste. Resources, Conservation \& Recycling. pp. 15-23.

Haidt, J., Rozin, P., McCauley, C., \& Imada, S. (1997). Body, psyche, and culture: The relationship between disgust and morality. Psychology and Developing Societies. 9(1), 107-131.

Hamdan, Saeed (1990). Social change in the Saudi family. Doctoral thesis. Iowa State University (United States).

Hebrok, M. and Heidenstrøm, N. (2019). Contextualising Food Waste Prevention-Decisive Moments within Everyday Practices. Journal of Cleaner Production. 210, 1435-1448. 
IOC (2006). Country Profile: Saudi Arabia. Library of Congress - Federal Research Division. Retrieved from http://www.loc.gov/rr/frd/cs/profiles.html. [accessed 09/07/2020]

Koivupuro, H. K., Hartikainen, H., Silvennoinen, K., Katajajuuri, J. M., Heikintalo, N., Reinikainen, A., \& Jalkanen, L. (2012). Influence of socio-demographical, behavioural and attitudinal factors on the amount of avoidable food waste generated in Finnish households. International journal of consumer studies, 36(2), 183-191.

Lazell, J. (2016). Consumer Food Waste Behaviour in Universities: Sharing as a Means of Prevention. Journal of Consumer Behaviour.15 (5), 430-439.

Magnusson, E., \& Marecek, J. (2015). Doing interview-based qualitative research: A learner's guide. Cambridge University Press.

Mattar, L., Abiad, M. G., Chalak, A., Diab, M., \& Hassan, H. (2018). Attitudes and behaviors shaping household food waste generation: Lessons from Lebanon. Journal of Cleaner Production. 198, 12191223.

Metz, H. C. (1992). ed. Saudi Arabia: A country Study. Washington: GPO for the Library of Congress. Nyrop, R. F. (1977). Area Handbook for Saudi Arabia (Vol. 51). Department of Defense, Department of the Army.

Porpino, G., Parente, J., \& Wansink, B. (2015). Food waste paradox: antecedents of food disposal in low income households. International journal of consumer studies, 39(6), 619-629.

Porpino, G., Wansink, B. \& Parente, J. (2016). Wasted Positive Intentions: The Role of Affection and Abundance on Household Food Waste. Journal of Food Products Marketing. 22 (7), pp. 733-751.

Quested, T. E., Marsh, E., Stunell, D., \& Parry, A. D. (2013). Spaghetti soup: The complex world of food waste behaviours. Resources, Conservation and Recycling. 79, 43-51.

Rolls, B. (1986). Sensory specific satiety. Nutrition Reviews. 44(3), 93e101.

Romani, S., Grappi, S., Bagozzi, R. P., \& Barone, A. M. (2018). Domestic food practices: A study of food management behaviors and the role of food preparation planning in reducing waste. Appetite. 121, 215-227.

Rozin, P., \& Fallon, A. E. (1987). A perspective on disgust. Psychological review. 94(1), 23.

Rubin, H. J., \& Rubin, I. S. (2012). Qualitative interviewing: The art of hearing data. sage.

Saudi Grains Organization (SAGO) (2019). Baseline: Food waste and loss index in the Kingdom of Saudi Arabia. Riyadh.

Schatzki, T.R. (2002). The site of the social: A philosophical account of the constitution of social life and change. Penn State Press

Soma, T. (2016). The tale of the crying rice: the role of unpaid foodwork and learning in food waste prevention and reduction in Indonesian households. In: J. Sumner, ed. Learning, food and sustainability: sites for resistance and change. New York: Palgrave Macmillan US, 19-34.

Soma, T. (2017). Gifting, ridding and the "everyday mundane": the role of class and privilege in food waste generation in Indonesia. Local Environment. 22 (12), pp. 1444-1460.

Southerton, D., \& Yates, L. (2015). Exploring food waste through the lens of social practice theories. In Waste management and sustainable consumption. ROUTLEDGE in association with GSE Research.

Stancu, V., Haugaard, P., \& Lähteenmäki, L. (2016). Determinants of consumer food waste behaviour: Two routes to food waste. Appetite. 96, 7-17.

Urrutia, I., Dias, G. M., \& Clapp, J. (2019). Material and visceral engagements with household food waste: Towards opportunities for policy interventions. Resources, Conservation and Recycling, 150, 104435.

Waitt, G. \& Phillips, C. (2016). Food waste and domestic refrigeration: a visceral and material approach. Social and Cultural Geography. 17 (3), pp. 359-379.

Warde, A. (2005). Consumption and theories of practice. Journal of consumer culture, 5(2), 131-153. Watson, M. \& Meah, A. (2013). Food, Waste And Safety: Negotiating Conflicting Social Anxieties Into The Practices Of Domestic Provisioning. Sociological Review. 60 (SUPPL.2), pp. 102-120.

Williams, H., Wikström, F., Otterbring, T., Löfgren, M., \& Gustafsson, A. (2012). Reasons for household food waste with special attention to packaging. Journal of cleaner production, 24, 141-148.

WRAP, (2008). The food we waste. United Kingdom 's Waste and Resources Action Programme (WRAP). Banbury. ISBN: 1-84405-383-0

WRAP, (2014). Household food and drink waste: A product focus. United Kingdom 's Waste and Resources Action Programme (WRAP). Banbury. ISBN: 978-1-84405-469-5

Yoreh, T., \& Scharper, S. (2020). Food waste, religion, and spirituality: Jewish, Christian and Muslim Approaches. In Reynolds, C., Soma, T., Spring, C., Lazell, J. Routledge Handbook of Food Waste 
